PROCEEDINGS OF THE

AMERICAN MATHEMATICAL SOCIETY

Volume 139, Number 8, August 2011, Pages 2903-2910

S 0002-9939(2010)10725-6

Article electronically published on November 2, 2010

\title{
BUSER'S ISOPERIMETRIC INEQUALITIES WITH INTEGRAL NORMS OF RICCI CURVATURE
}

\author{
SEONG-HUN PAENG
}

(Communicated by Jianguo Cao)

\begin{abstract}
We generalize Buser's isoperimetric inequality with integral norms of Ricci curvature.
\end{abstract}

\section{INTRODUCTION}

The isoperimetric inequality is one of the most important topics in geometry. In Riemannain geometry, several isoperimetric inequalities have been proved under curvature pinching conditions. Buser's isoperimetric inequality is as follows $\mathrm{B}$, Ch: Let $M$ be an $n$-dimensional complete Riemannian manifold and $B(x, r)$ be the $r$-ball in $M$ centered at $x$ for $r>0$. We denote the $(n-1)$-volume of a hypersurface $\Gamma$ by $A(\Gamma)$ and $\min \left\{\operatorname{vol}\left(D_{1}\right), \operatorname{vol}\left(D_{2}\right)\right\}$ by $V\left(D_{1}, D_{2}\right)$ for disjoint open sets $D_{1}, D_{2}$.

Theorem 1.1. If the Ricci curvature satisfies that $\operatorname{Ric}_{M} \geq(n-1) \lambda$ for $\lambda \leq 0$, then there exists a positive constant $c(n, \lambda, r)$ depending on $n, \lambda, r$ such that for any $x \in M$, a dividing smooth hypersurface $\Gamma$ in $B(x, r)$ with $\bar{\Gamma}$ embedded in $\overline{B(x, r)}$ and $B(x, r) \backslash \Gamma=D_{1} \cup D_{2}$, we have

$$
A(\Gamma) \geq c(n, \lambda, r) V\left(D_{1}, D_{2}\right) .
$$

Recently, there have been many attempts to replace curvature bounds with integral norms of curvature. In Ga, Gallot obtained an isoperimetric inequality with integral norms of Ricci curvature. He obtain a lower bound of $A(\partial \Omega)$ with $V(\Omega, M \backslash \Omega)$ and a condition on the integral norm of Ricci curvature. Note that $\Gamma$ does not need to be $\partial \Omega$ in the case of Buser's isoperimetric inequality.

In [PW], Petersen and Wei generalized the Bishop-Gromov volume comparison estimate with integral norms of Ricci curvature. In this paper, we generalize Buser's isoperimetric inequalities with integral norms of Ricci curvature by using the volume comparison in [PW].

First, we define the following notation for the integral norm of Ricci curvature. Let $g(x)$ be the smallest eigenvalue of the Ricci tensor at $x \in M$ and $u_{+}=\max (0, u)$

Received by the editors March 25, 2010 and, in revised form, April 18, 2010.

2000 Mathematics Subject Classification. Primary 53C20.

Key words and phrases. Buser's isoperimetric inequality, integral norm of Ricci curvature.

This work was supported by Konkuk University and National Research Foundation of Korea (NRF) grant funded by the Korea Government (MEST) (No. 2010-0015791 and 2010-0001194). 
be the positive part of $u$. For $2 p>n, \lambda \leq 0$, we define $k_{x}(\lambda, p, R)$ as follows:

$$
k_{x}(\lambda, p, R)=\int_{B(x, R)}\left((-g(x)+(n-1) \lambda)_{+}\right)^{p} d v \text { for } x \in M .
$$

If $\operatorname{Ric}_{M} \geq \lambda$, then $k_{x}(\lambda, p, R)=0$.

We will prove the following isoperimetric inequalities:

Theorem 1.2. If $B(x, r)$ is a convex $r$-ball and $k_{x}(\lambda, p, r) \leq K$ for $x \in M$, then there exist positive constants $c_{1}(n, \lambda, r)$ depending on $n, \lambda, r$ and $c_{2}(n, p, \lambda, r, K)$ depending on $n, p, \lambda, r, K$ such that for a dividing smooth hypersurface $\Gamma$ in $B(x, r)$ with $\bar{\Gamma}$ embedded in $\overline{B(x, r)}$ and $B(x, r) \backslash \Gamma=D_{1} \cup D_{2}$, we have

$$
A(\Gamma) \geq c_{1}(n, \lambda, r) V\left(D_{1}, D_{2}\right)-c_{2}(n, p, \lambda, r, K),
$$

where $c_{2}(n, p, \lambda, r, K) \rightarrow 0$ as $K \rightarrow 0$.

We can obtain $c_{1}, c_{2}$ explicitly. In $\mathrm{Ch}$, Theorem 1.1 is proved for a more general domain, i.e. a star-shaped domain $D$ with $B(x, r / 2) \subset D \subset B(x, r)$. We can also prove Theorem 1.2 for a convex domain $D$ with $B(x, r / 2) \subset D \subset B(x, r)$.

The boundary rigidity problem is to what extent a Riemannian metric on a compact manifold with boundary is determined from the distances between boundary points $[\mathrm{C},[\mathrm{S}$. Linearizing the boundary rigidity problem, the integral geometry problem is to what extent is a symmetric tensor field determined by the set of integrals along geodesics connecting boundary points $[\mathrm{S}$. In $[\mathrm{Pa}$, an upper bound of the volume entropy and the simplicial volume with integral norms of Ricci curvature over closed geodesics are obtained. It is also interesting to consider Buser's isoperimetric problem with integral norms of curvature over all geodesics connecting boundary points.

For a geodesic segment $\gamma$, define $k_{\gamma}$ for $p>1$ as follows:

$$
k_{\gamma}(p)=\int_{\gamma} \max \left\{0,-\operatorname{Ric}\left(\gamma^{\prime}, \gamma^{\prime}\right)\right\}^{p} .
$$

Let $G(x, r)$ be the set of geodesic segments in $B(x, r)$ connecting two points in $\partial B(x, r)$.

Theorem 1.3. If $B(x, r)$ is a convex $r$-ball and $k_{\gamma}(p) \leq k$ for any $\gamma \in G(x, r)$, then there exists a positive constant $c_{3}(n, p, k, r)$ depending on $n, p, k, r$ such that for a dividing smooth hypersurface $\Gamma$ in $B(x, r)$ with $\bar{\Gamma}$ embedded in $\overline{B(x, r)}$ and $B(x, r) \backslash \Gamma=D_{1} \cup D_{2}$, we have

$$
A(\Gamma) \geq c_{3}(n, p, k, r) V\left(D_{1}, D_{2}\right) .
$$

Note that in this theorem, there are no additional terms such as $c_{2}(n, p, \lambda, r, K)$ in Theorem 1.2. Also $c_{3}$ can be obtained explicitly.

\section{Proof of Theorem 1.2}

We will follow the proof of Buser's isoperimetric inequality in $\mathrm{Ch}$. Around $x$, use exponential polar coordinates and write the volume element as $d \mathrm{vol}=\omega d t \wedge d \theta$ inside the cut locus, where $d \theta$ is the standard volume element on the unit sphere $S^{n-1} \cong U_{x} M$, where $U_{x} M$ is the unit tangent space of $x$. Sometimes we abbreviate $\omega(t, \theta)$ to $\omega(t)$. Outside the cut locus, $\omega=0$. Let $\omega_{\lambda}$ be the $\omega$ for the $n$-dimensional space form $M_{\lambda}^{n}$ with constant curvature $\lambda$. Let $V_{\lambda}(s)=\int_{0}^{s} \omega_{\lambda}(t) d t$. We know that 
$\omega^{\prime}=h \omega$, where $h$ is the mean curvature of the distance spheres around $x$ and $h$, satisfies

$$
h^{\prime}+\frac{h^{2}}{n-1} \leq-\operatorname{Ric}\left(\partial_{t}, \partial_{t}\right)
$$

where $\partial_{t}$ is the unit gradient of the distance function $d(\cdot, x)$.

The Bishop-Gromov volume comparison theorem implies that $\frac{d}{d s} \frac{\omega(s, \theta)}{\omega_{\lambda}(s)} \leq 0$ if $\operatorname{Ric}_{M} \geq(n-1) \lambda$. Then we have

$$
\frac{\omega(l, \theta)}{\omega_{\lambda}(l)} \geq \frac{1}{V_{\lambda}(s)-V_{\lambda}(l)} \int_{l}^{s} \omega(t, \theta) d t
$$

for $l \leq s$ and

$$
\frac{1}{V_{\lambda}\left(r_{1}\right)-V_{\lambda}\left(r_{0}\right)} \int_{r_{0}}^{r_{1}} \omega(s, \theta) d s \geq \frac{1}{V_{\lambda}\left(r_{2}\right)-V_{\lambda}\left(r_{1}\right)} \int_{r_{1}}^{r_{2}} \omega(s, \theta) d s
$$

for $r_{0} \leq r_{1} \leq r_{2} \leq r$.

With integral norms of Ricci curvature, we obtain the following comparisons. Let $\omega_{\lambda}^{\prime}=h_{\lambda} \omega$ and define $\psi=\psi(s, \cdot)=\max \left\{0, h(s, \cdot)-h_{\lambda}(s, \cdot)\right\}$. Then we have

$$
\frac{d}{d s} \frac{\omega}{\omega_{\lambda}} \leq \psi \frac{\omega}{\omega_{\lambda}} .
$$

Integrating the above, for $r_{1} \leq s$, we obtain that

$$
\frac{\omega(s)}{\omega_{\lambda}(s)}-\frac{\omega\left(r_{1}\right)}{\omega_{\lambda}\left(r_{1}\right)} \leq \int_{r_{1}}^{s} \psi \frac{\omega}{\omega_{\lambda}} \leq \frac{1}{\omega_{\lambda}\left(r_{1}\right)} \int_{r_{1}}^{s} \psi \omega .
$$

Then we have for $r_{2}>r_{1}$,

$$
\begin{aligned}
\int_{r_{1}}^{r_{2}} \omega(s) d s & \leq\left(\frac{\omega\left(r_{1}\right)}{\omega_{\lambda}\left(r_{1}\right)}+\frac{1}{\omega_{\lambda}\left(r_{1}\right)} \int_{r_{1}}^{r_{2}} \psi \omega\right) \int_{r_{1}}^{r_{2}} \omega_{\lambda}(s) d s \\
& \leq \frac{V_{\lambda}\left(r_{2}\right)-V_{\lambda}\left(r_{1}\right)}{\omega_{\lambda}\left(r_{1}\right)}\left(\omega\left(r_{1}\right)+\int_{r_{1}}^{r_{2}} \psi \omega\right),
\end{aligned}
$$

So

$$
\frac{\int_{r_{1}}^{r_{2}} \omega(s) d s}{V_{\lambda}\left(r_{2}\right)-V_{\lambda}\left(r_{1}\right)} \leq \frac{\omega\left(r_{1}\right)}{\omega_{\lambda}\left(r_{1}\right)}+\frac{1}{\omega_{\lambda}\left(r_{1}\right)} \int_{r_{1}}^{r_{2}} \psi \omega
$$

For $r_{0} \leq l \leq r_{1} \leq r_{2} \leq r$,

$$
\begin{aligned}
\frac{\int_{r_{1}}^{r_{2}} \omega(s) d s}{V_{\lambda}\left(r_{2}\right)-V_{\lambda}\left(r_{1}\right)} & \leq \frac{\omega\left(r_{1}\right)}{\omega_{\lambda}\left(r_{1}\right)}+\frac{1}{\omega_{\lambda}\left(r_{1}\right)} \int_{r_{1}}^{r_{2}} \psi \omega \\
& \leq \frac{\omega(l)}{\omega_{\lambda}(l)}+\frac{1}{\omega_{\lambda}(l)} \int_{l}^{r_{1}} \psi \omega+\frac{1}{\omega_{\lambda}\left(r_{1}\right)} \int_{r_{1}}^{r_{2}} \psi \omega \\
& \leq \frac{\omega(l)}{\omega_{\lambda}(l)}+\frac{2}{\omega_{\lambda}(l)} \int_{0}^{r} \psi \omega .
\end{aligned}
$$

Then we have

$$
\frac{\int_{r_{1}}^{r_{2}} \omega(s) d s}{V_{\lambda}\left(r_{2}\right)-V_{\lambda}\left(r_{1}\right)} \leq \frac{\int_{r_{0}}^{r_{1}} \omega(s) d s}{V_{\lambda}\left(r_{1}\right)-V_{\lambda}\left(r_{0}\right)}+\frac{2 r}{V_{\lambda}\left(r_{1}\right)-V_{\lambda}\left(r_{0}\right)} \int_{0}^{r} \psi \omega
$$

From now on, we follow the proof of Theorem 1.1 in [Ch] with (2.6) and (2.8) instead of (2.2) and (2.3). 
Fix $t \in(0, r / 2)$. We set $B_{s}=B(x, s), V_{s}=\operatorname{vol}(B(x, s))$. We may assume that $\operatorname{vol}\left(D_{1} \cap B_{r / 2}\right) \leq V_{r / 2} / 2$. Fix any $\alpha \in(0,1), t<r / 2$. We consider two cases. The first is $\operatorname{vol}\left(D_{1} \cap B_{r / 2}\right) \leq \alpha \operatorname{vol}\left(D_{1}\right)$ and the second is $\operatorname{vol}\left(D_{1} \cap B_{r / 2}\right) \geq \alpha \operatorname{vol}\left(D_{1}\right)$.

Case 1. Let $C(x)$ be the cut locus of $x$. For $p \in D_{1} \backslash C(x)$, let $p^{*}$ be the first point on the geodesic segment $p x$ from $p$ to $x$ where $p x$ intersects $\Gamma$. If $p x \subset D_{1}$, then $p^{*}=x$. We define the subsets $A_{1}, A_{2}, A_{3}$ of $D_{1}$ as follows:

$$
\begin{aligned}
& A_{1}=\left\{p \in D_{1} \backslash\left(C(x) \cup \overline{B_{r / 2}}\right) \mid p^{*} \notin \overline{B_{t}}\right\}, \\
& A_{2}=\left\{p \in D_{1} \backslash\left(C(x) \cup \overline{B_{r / 2}}\right) \mid p^{*} \in \overline{B_{t}}\right\}, \\
& A_{3}=\left(B_{r / 2} \backslash B_{t}\right) \cap \bigcup_{p \in A_{2}}\left\{\exp _{x} \tau \theta \mid t \leq \tau \leq s, \text { where } p=\exp _{x} s \theta\right\} .
\end{aligned}
$$

It will be helpful to refer to Figure $6.1 \mathrm{in}$ Ch.

We define $\rho=\rho(t, \cdot)=\max \left\{0,(n-1) \lambda-\operatorname{Ric}\left(\partial_{t}, \partial_{t}\right)\right\}$. From Lemma 2.2 in [PW], for a subset $S$ of $U_{x} M$, we have

$$
\begin{aligned}
\int_{S} \int_{0}^{r} \psi \omega & \leq\left(\int_{S^{n-1}} \int_{0}^{r} \psi^{2 p} \omega\right)^{\frac{1}{2 p}}\left(\int_{S^{n-1}} \int_{0}^{r} \omega\right)^{1-\frac{1}{2 p}} \\
& \leq c_{1}(n, p) \operatorname{vol}(B(x, r))^{1-\frac{1}{2 p}}\left(\int_{S^{n-1}} \int_{0}^{r} \rho^{p} \omega\right)^{\frac{1}{2 p}} \\
& \leq c_{1}(n, p) \operatorname{vol}(B(x, r))^{1-\frac{1}{2 p}} k_{x}(\lambda, p, r)^{\frac{1}{2 p}}
\end{aligned}
$$

for some constant $c_{1}(n, p)>0$. By (2.8) and (2.10), we obtain that

$$
\begin{aligned}
\operatorname{vol}\left(A_{2}\right) \leq & \frac{V_{\lambda}(r)-V_{\lambda}\left(\frac{r}{2}\right)}{V_{\lambda}\left(\frac{r}{2}\right)-V_{\lambda}(t)} \operatorname{vol}\left(A_{3}\right) \\
& +\frac{2 r c_{1}(n, p) \operatorname{vol}(B(x, r))^{1-\frac{1}{2 p}} K^{\frac{1}{2 p}}\left(V_{\lambda}(r)-V_{\lambda}\left(\frac{r}{2}\right)\right)}{V_{\lambda}\left(\frac{r}{2}\right)-V_{\lambda}(t)} .
\end{aligned}
$$

By [PW], we have $\operatorname{vol}(B(x, r)) \leq v(n, p, \lambda, r, K)$ for some constant $v(n, p, \lambda, r, K)$ depending on $n, p, \lambda, r, K$. Let

$$
\begin{aligned}
\gamma_{1} & =\frac{V_{\lambda}(r)-V_{\lambda}\left(\frac{r}{2}\right)}{V_{\lambda}\left(\frac{r}{2}\right)-V_{\lambda}(t)} \\
\gamma_{2} & =\frac{2 c_{1}(n, p) v(n, p, \lambda, r, K)^{1-\frac{1}{2 p}} K^{\frac{1}{2 p}}\left(V_{\lambda}(r)-V_{\lambda}\left(\frac{r}{2}\right)\right)}{V_{\lambda}\left(\frac{r}{2}\right)-V_{\lambda}(t)} \\
& =2 c_{1}(n, p) v(n, p, \lambda, r, K)^{1-\frac{1}{2 p}} K^{\frac{1}{2 p}} \gamma_{1} .
\end{aligned}
$$

Then we obtain that

$$
\operatorname{vol}\left(A_{2}\right) \leq \gamma_{1} \operatorname{vol}\left(A_{3}\right)+r \gamma_{2} .
$$

Since $(1-\alpha) \operatorname{vol}\left(D_{1}\right) \leq \operatorname{vol}\left(D_{1} \backslash B_{r / 2}\right)=\operatorname{vol}\left(A_{1}\right)+\operatorname{vol}\left(A_{2}\right)$ and $\operatorname{vol}\left(A_{3}\right) \leq \operatorname{vol}\left(D_{1} \cap\right.$ $\left.B_{r / 2}\right) \leq \alpha \operatorname{vol}\left(D_{1}\right)$, we have

$$
\left(1-\alpha\left(1+\gamma_{1}\right)\right) \operatorname{vol}\left(D_{1}\right) \leq \operatorname{vol}\left(A_{1}\right)+r \gamma_{2} .
$$

Now we only need to compute an upper bound of $\operatorname{vol}\left(A_{1}\right)$. Let $\left\{\exp _{x}(t \theta)\right\} \cap A_{1}=$ $\bigcup_{j_{\theta}} \exp _{x}\left[\alpha_{j_{\theta}}^{\prime}, \beta_{j_{\theta}}\right]$. We set $\alpha_{j_{\theta}}=\alpha_{j_{\theta}}^{\prime}$ if $\alpha_{j_{\theta}}^{\prime}>r / 2$ and $\alpha_{j_{\theta}}=\left|\exp _{x}^{-1}\left(\exp _{x}(r / 2) \theta\right)^{*}\right|$ if $\alpha_{j_{\theta}}^{\prime}=\frac{r}{2}$, i.e. $\exp _{x} \alpha_{j_{\theta}} \in \Gamma$. 
With (2.6), for $t<r / 2 \leq \alpha_{j_{\theta}} \leq s \leq \beta_{j_{\theta}} \leq r$, we obtain that

$$
\frac{\int_{\alpha_{j_{\theta}}}^{\beta_{j_{\theta}}} \omega(s) d s}{V_{\lambda}\left(\beta_{j_{\theta}}\right)-V_{\lambda}\left(\alpha_{j_{\theta}}\right)} \leq \frac{\omega\left(\alpha_{j_{\theta}}\right)}{\omega_{\lambda}\left(\alpha_{j_{\theta}}\right)}+\frac{1}{\omega_{\lambda}\left(\alpha_{j_{\theta}}\right)} \int_{\alpha_{j_{\theta}}}^{\beta_{j_{\theta}}} \psi \omega .
$$

Let $\nu$ be the projection to $U_{x} M$ such that $\nu\left(\exp _{x} s \theta\right)=\theta$ and let $S$ be the subset $\nu\left(A_{1}\right)$ of $U_{x} M$. By (2.14),

$$
\begin{gathered}
\int_{S} \sum_{j_{\theta}} \int_{\alpha_{j_{\theta}}}^{\beta_{j_{\theta}}} \omega(s) d s d \theta \leq \frac{V_{\lambda}(r)-V_{\lambda}(t)}{\omega_{\lambda}(t)}\left(\int_{S} \sum_{j_{\theta}} \omega\left(\alpha_{j_{\theta}}\right) d \theta\right. \\
\left.+\int_{S} \sum_{j_{\theta}} \int_{\alpha_{j_{\theta}}}^{\beta_{j_{\theta}}} \psi \omega d \theta\right) .
\end{gathered}
$$

We have

$$
\begin{aligned}
\int_{S} \sum_{j_{\theta}} \int_{\alpha_{j_{\theta}}}^{\beta_{j_{\theta}}} \psi \omega d \theta & \leq\left(\int_{S} \sum_{j_{\theta}} \int_{\alpha_{j_{\theta}}}^{\beta_{j_{\theta}}} \psi^{2 p} \omega d \theta d t\right)^{\frac{1}{2 p}}\left(\int_{S} \sum_{j_{\theta}} \int_{\alpha_{j_{\theta}}}^{\beta_{j_{\theta}}} \omega d \theta d t\right)^{1-\frac{1}{2 p}} \\
& \leq\left(\int_{S} \int_{0}^{r} \psi^{2 p} \omega d \theta d t\right)^{\frac{1}{2 p}}\left(\int_{S} \int_{0}^{r} \omega d \theta d t\right)^{1-\frac{1}{2 p}}
\end{aligned}
$$

From the definition of $p^{*}$, we have that

$$
\left(\int_{S} \int_{0}^{r} \omega d \theta d t\right)^{1-\frac{1}{2 p}} \leq \operatorname{vol}(B(x, r))^{1-\frac{1}{2 p}} \leq v(n, p, \lambda, r, K)^{1-\frac{1}{2 p}}
$$

As in (2.10), we have

$$
\int_{S} \sum_{j_{\theta}} \int_{\alpha_{j_{\theta}}}^{\beta_{j_{\theta}}} \psi \omega d \theta \leq c_{1}(n, p) K^{\frac{1}{2 p}} v(n, p, \lambda, r, K)^{1-\frac{1}{2 p}} .
$$

Then we obtain that

$$
\begin{aligned}
\operatorname{vol}\left(A_{1}\right) & =\int_{S} \sum_{j_{\theta}} \int_{\alpha_{j_{\theta}}}^{\beta_{j_{\theta}}} \omega(s) d s d \theta \\
& \leq \frac{V_{\lambda}(r)-V_{\lambda}(t)}{\omega_{\lambda}(t)}\left(\int_{S} \sum_{j_{\theta}} \omega\left(\alpha_{j_{\theta}}\right) d \theta+c_{1}(n, p) K^{\frac{1}{2 p}} v(n, p, \lambda, r, K)^{1-\frac{1}{2 p}}\right) \\
& \leq \frac{V_{\lambda}(r)-V_{\lambda}(t)}{\omega_{\lambda}(t)}\left(A(\Gamma)+c_{1}(n, p) K^{\frac{1}{2 p}} v(n, p, \lambda, r, K)^{1-\frac{1}{2 p}}\right) .
\end{aligned}
$$

So we obtain that

$$
\begin{aligned}
A(\Gamma) & \geq \frac{\omega_{\lambda}(t)}{V_{\lambda}(r)-V_{\lambda}(t)} \operatorname{vol}\left(A_{1}\right)-c_{1}(n, p) K^{\frac{1}{2 p}} v(n, p, \lambda, r, K)^{1-\frac{1}{2 p}} \\
& \geq \frac{\omega_{\lambda}(t)}{V_{\lambda}(r)-V_{\lambda}(t)} \operatorname{vol}\left(A_{1}\right)-c_{1}(n, p) K^{\frac{1}{2 p}} v(n, p, \lambda, r, K)^{1-\frac{1}{2 p}} .
\end{aligned}
$$

From the definition (2.12), we have

$$
c_{1}(n, p) K^{\frac{1}{2 p}} v(n, p, \lambda, r, K)^{1-\frac{1}{2 p}}=\frac{\gamma_{2}}{2 \gamma_{1}} .
$$


Since $\operatorname{vol}\left(A_{1}\right) \geq\left(1-\alpha\left(1+\gamma_{1}\right)\right) \operatorname{vol}\left(D_{1}\right)-r \gamma_{2}$ and $v(n, p, \lambda, r, K) \geq \operatorname{vol}(B(x, r))$, we obtain from (2.21) that

$$
\begin{aligned}
A(\Gamma) \geq & \left(1-\alpha\left(1+\gamma_{1}\right)\right) \frac{\omega_{\lambda}(t)}{V_{\lambda}(r)-V_{\lambda}(t)} \operatorname{vol}\left(D_{1}\right)-\frac{r \gamma_{2} \omega_{\lambda}(t)}{V_{\lambda}(r)-V_{\lambda}(t)} \\
& \quad-c_{1}(n, p) K^{\frac{1}{2 p}} v(n, p, \lambda, r, K)^{1-\frac{1}{2 p}} \\
\geq & \left(1-\alpha\left(1+\gamma_{1}\right)\right) \frac{\omega_{\lambda}(t)}{V_{\lambda}(r)-V_{\lambda}(t)} \operatorname{vol}\left(D_{1}\right)-\frac{r \gamma_{2} \omega_{\lambda}(t)}{V_{\lambda}(r)-V_{\lambda}(t)}-\frac{\gamma_{2}}{2 \gamma_{1}} .
\end{aligned}
$$

Case 2. In Case 2, we consider another exponential map $\exp _{w_{0}}$ centered at $w_{0}$ instead of $\exp _{x}$ to compute the volume, where $w_{0}$ is determined by the following lemma:

Lemma 2.1. Set either $W_{0}=D_{1} \cap B_{r / 2}, W_{1}=D_{2} \cap B_{r / 2}$ or $W_{0}=D_{2} \cap B_{r / 2}$, $W_{1}=D_{1} \cap B_{r / 2}$. Then for at least one of two choices of the pair $\left\{W_{0}, W_{1}\right\}$, we have the existence of a point $w_{0} \in W_{0}$ and $\mathcal{W}_{1} \subset W_{1}$ such that

(i) $\operatorname{vol}\left(\mathcal{W}_{1}\right) \geq \operatorname{vol}\left(W_{1}\right) / 2$;

(ii) for $q \in \mathcal{W}_{1}$, every minimizing directed geodesic segment qw from $q$ to $w_{0}$ intersects $\Gamma$ in a first point $q^{*}$ such that $d\left(q, q^{*}\right) \leq d\left(q^{*}, w_{0}\right)$.

For Case 2, $\omega(t, \theta)$ is the volume element of $\partial B\left(w_{0}, t\right)$ for $\exp _{w_{0}}$. Let $\nu_{1}$ : $B\left(x, \frac{r}{2}\right) \backslash C\left(w_{0}\right) \rightarrow U_{w_{0}} M$ be a projection as described before for $x$. To each $\theta \in \nu_{1}\left(\mathcal{W}_{1} \backslash C\left(w_{0}\right)\right)$, we determine a collection of disjoint intervals $\left\{\left(\alpha_{j_{\theta}}, \beta_{j_{\theta}}\right)\right\}$ as follows: For $q=\exp _{w_{0}} t_{0} \theta \in \mathcal{W}_{1}, \alpha_{q}=d\left(w_{0}, q^{*}\right)$ and $\beta_{q}=\sup \left\{t>\alpha_{q} \mid \exp _{w_{0}} t \theta \in\right.$ $\left.\mathcal{W}_{1}, \quad\left\{\exp _{w_{0}} s \theta \mid s \in\left(\alpha_{q}, t\right)\right\} \subset B(x, r) \backslash \Gamma\right\}$. Then $\mathcal{W}_{1} \cap\left\{\exp _{w_{0}} t \theta\right\} \subset$ $\bigcup_{j_{\theta}}\left\{\exp _{w_{0}} s \theta \mid s \in\left(\alpha_{j_{\theta}}, \beta_{j_{\theta}}\right)\right\}$ and

$$
\beta_{j_{\theta}} \leq 2 \alpha_{j_{\theta}}<r
$$

Similarly as in (2.19), we have by (2.23),

$$
\begin{aligned}
\operatorname{vol}\left(\mathcal{W}_{1}\right) & \leq \int_{\nu_{1}\left(\mathcal{W}_{1} \backslash C\left(w_{0}\right)\right.} d \theta \sum_{j_{\theta}} \int_{\alpha_{j_{\theta}}}^{\beta_{j_{\theta}}} \omega(s) d s \\
& \leq \frac{V_{\lambda}(r)-V_{\lambda}\left(\frac{r}{2}\right)}{\omega_{\lambda}\left(\frac{r}{2}\right)}\left(A(\Gamma)+c_{1}(n, p) K^{\frac{1}{2 p}} v(n, p, \lambda, r, K)^{1-\frac{1}{2 p}}\right) .
\end{aligned}
$$

By (2.21) and $\alpha \operatorname{vol}\left(D_{1}\right) \leq 2 \operatorname{vol}\left(\mathcal{W}_{1}\right)$,

$$
\begin{aligned}
A(\Gamma) & \geq \frac{\omega_{\lambda}\left(\frac{r}{2}\right)}{V_{\lambda}(r)-V_{\lambda}\left(\frac{r}{2}\right)} \operatorname{vol}\left(\mathcal{W}_{1}\right)-c_{1}(n, p) K^{\frac{1}{2 p}} v(n, p, \lambda, r, K)^{1-\frac{1}{2 p}} \\
& \geq \frac{\omega_{\lambda}\left(\frac{r}{2}\right)}{V_{\lambda}(r)-V_{\lambda}\left(\frac{r}{2}\right)} \operatorname{vol}\left(\mathcal{W}_{1}\right)-\frac{\gamma_{2}}{2 \gamma_{1}} \\
& \geq \frac{\alpha}{2} \frac{\omega_{\lambda}\left(\frac{r}{2}\right)}{V_{\lambda}(r)-V_{\lambda}\left(\frac{r}{2}\right)} \operatorname{vol}\left(D_{1}\right)-\frac{\gamma_{2}}{2 \gamma_{1}} .
\end{aligned}
$$

Simply if $t=\frac{r}{4}$ and $\alpha=\frac{1}{2\left(1+\gamma_{1}\right)}$ for $t=\frac{r}{4}$, then we obtain that

$$
c_{1}(n, \lambda, r)=\min \left(\frac{1}{4\left(1+\gamma_{1}\right)} \frac{\omega_{\lambda}\left(\frac{r}{2}\right)}{V_{\lambda}(r)-V_{\lambda}\left(\frac{r}{2}\right)}, \frac{1}{2} \frac{\omega_{\lambda}\left(\frac{r}{4}\right)}{V_{\lambda}(r)-V_{\lambda}\left(\frac{r}{4}\right)}\right)
$$


and

$$
c_{2}(n, p, \lambda, r, K)=\frac{\gamma_{2}}{2 \gamma_{1}}+\frac{r \gamma_{2} \omega_{\lambda}\left(\frac{r}{4}\right)}{V_{\lambda}(r)-V_{\lambda}\left(\frac{r}{4}\right)},
$$

although these coefficients are not optimized. Since $\gamma_{2} \rightarrow 0$ as $K \rightarrow 0$, we obtain that $c_{2}(n, p, \lambda, r, K) \rightarrow 0$ as $K \rightarrow 0$. Since $c_{1}(n, p)$ and $v(n, p, \lambda, r, K)$ are obtained explicitly in [PW], we can obtain $c_{1}, c_{2}$ explicitly, too.

\section{Proof of Theorem 1.3}

We denote $\frac{n-1}{t}$ by $h_{0}(t)$. Then $h_{0}^{\prime}+\frac{h_{0}^{2}}{n-1}=0$. Let $\psi(t, \theta)=\max \{0, h(t, \theta)-$ $\left.h_{0}(t)\right\}$. Since $\frac{\omega^{\prime}}{\omega}=h$, integrating this equation, we obtain that

$$
\log \left(\frac{\omega\left(r_{2}, \theta\right)}{\omega\left(r_{1}, \theta\right)}\right)=\int_{r_{1}}^{r_{2}} h d t \leq \int_{r_{1}}^{r_{2}} h_{0} d t+\int_{r_{1}}^{r_{2}} \psi d t .
$$

So we have

$$
\begin{aligned}
\omega\left(r_{2}, \theta\right) & \leq e^{\int_{r_{1}}^{r_{2}} \psi d t} e^{\int_{r_{1}}^{r_{2}} h_{0} d t} \omega\left(r_{1}, \theta\right) \\
& =\left(\frac{r_{2}}{r_{1}}\right)^{n-1} e^{\int_{r_{1}}^{r_{2}} \psi d t} \omega\left(r_{1}, \theta\right) .
\end{aligned}
$$

By $[\mathrm{Pa}$, for any $p>1$, we have

$$
\int_{0}^{s} \psi^{2 p} d t \leq(n-1)^{p} \int_{0}^{s} \rho^{p} d t
$$

so for $0<r_{1} \leq r_{2} \leq r$,

$$
\begin{aligned}
\int_{r_{1}}^{r_{2}} \psi d t & \leq \int_{0}^{r} \psi d t \leq \sqrt{n-1} r^{1-\frac{1}{2 p}}\left(\int_{0}^{r} \rho^{p} d t\right)^{\frac{1}{2 p}} \\
& \leq \sqrt{n-1} r^{1-\frac{1}{2 p}} k^{\frac{1}{2 p}} .
\end{aligned}
$$

Then for $r_{1}<r_{2}$,

$$
\omega\left(r_{2}\right) \leq C_{1}(n, p, k, r)\left(\frac{r_{2}}{r_{1}}\right)^{n-1} \omega\left(r_{1}\right)
$$

for some constant $C(n, p, k, r)$. So, for $r_{0} \leq l \leq r_{1}<r_{2}<r$,

$$
\begin{aligned}
\int_{r_{1}}^{r_{2}} \omega(s) d s & \leq C_{1}(n, p, k, r)\left(r_{2}-r_{1}\right)\left(\frac{r_{2}}{r_{1}}\right)^{n-1} \omega\left(r_{1}\right) \\
& \leq C_{1}(n, p, k, r)^{2}\left(r-r_{1}\right)\left(\frac{r}{r_{0}}\right)^{2(n-1)} \omega(l)
\end{aligned}
$$

and

$$
\int_{r_{1}}^{r_{2}} \omega(s) d s \leq C_{1}(n, p, k, r)^{2} \frac{r-r_{1}}{r_{1}-r_{0}}\left(\frac{r}{r_{0}}\right)^{2(n-1)} \int_{r_{0}}^{r_{1}} \omega(s) d s .
$$

Instead of (2.2), (2.3), we use (3.5), (3.6) and follow the proof of [Ch]. For Case 1, if we denote $C_{1}(n, p, k, r)^{2} \frac{r / 2}{r / 2-t}\left(\frac{r}{t}\right)^{2(n-1)}$ by $C_{2}(n, p, k, r, t)$, we have

$$
\frac{\operatorname{vol}\left(A_{2}\right)}{\operatorname{vol}\left(A_{3}\right)} \leq C_{2}(n, p, k, r, t)
$$


and by (3.5),

$$
\begin{aligned}
\operatorname{vol}\left(A_{1}\right) & =\int_{S} \sum_{j_{\theta}} \int_{\alpha_{j_{\theta}}}^{\beta_{j_{\theta}}} \omega(s) d s d \theta \\
& \leq C_{1}(n, p, k, r) r\left(\frac{r}{t}\right)^{n-1} \int_{S} \sum_{j_{\theta}} \omega\left(\alpha_{j_{\theta}}\right) d \theta \\
& \left.\left.\leq C_{1}(n, p, k, r) r\right) \frac{r}{t}\right)^{n-1} A(\Gamma) .
\end{aligned}
$$

For Case 2, we have $\beta_{j_{\theta}} \leq 2 \alpha_{j_{\theta}}$. With the first inequality in (3.5), we have

$$
\begin{aligned}
\int_{\alpha_{j_{\theta}}}^{\beta_{j_{\theta}}} \omega(s) d s & \leq C_{1}(n, p, k, r) r\left(\frac{\beta_{j_{\theta}}}{\alpha_{j_{\theta}}}\right)^{n-1} \omega\left(\alpha_{j_{\theta}}\right) \\
& \leq 2^{n-1} r C_{1}(n, p, k, r) \omega\left(\alpha_{j_{\theta}}\right) .
\end{aligned}
$$

Then

$$
\operatorname{vol}\left(\mathcal{W}_{1}\right) \leq 2^{n-1} r C_{1}(n, p, k, r) A(\Gamma) .
$$

Then we can prove Theorem 1.3 by the same arguments as $\mathrm{Ch}$.

\section{REFERENCES}

[B] P. Buser, A note on the isoperimetric constant, Ann. Sci. École Norm. Sup., Paris 15 (1982), 213-230. MR683635 (84e:58076)

[Ch] I. Chavel, Riemannian geometry-A modern introduction, Cambridge University Press (1993). MR.1271141 (95j:53001)

[C] C. B. Croke, Rigidity theorems in Riemannian geometry, Geometric methods in inverse problems and PDE control, The IMA volumes in Mathematics and its Applications, Springer (1993), 47-72. MR2169902 (2007c:53052)

[Ga] S. Gallot, Isoperimetric inequalities based on integral norms of Ricci curvature, Astérisque 157-158 (1988), 191-216. MR976219 (90a:58179)

[Pa] S.-H. Paeng, Volume entropy and integral Ricci curvatures over closed geodesics, Proc. Amer. Math. Soc. 135 (2007), 3677-3684. MR2336584 (2008g:53033)

[PW] P. Petersen, G. Wei, Relative volume comparison with integral curvature bounds, Geom. Funct. Anal. 7 (1997), 1031-1045. MR.1487753 (99c:53023)

[S] V. Sharafutdinov, Ray transform and some rigidity problems for Riemannian metrics, Geometric methods in inverse problems and PDE control, The IMA volumes in Mathematics and its Applications, Springer (1993), 215-238. MR2169905 (2006g:53052)

Department of Mathematics, Konkuk University, 1 Hwayang-dong, Gwanguin-gu, Seoul 143-701, Republic of Korea

E-mail address: shpaeng@konkuk.ac.kr 